\title{
Role of Family System, Positive Emotions and Resilience in Social Adjustment among Pakistani Adolescents
}

\author{
Najam-us-Sahar \\ Lecturer Department of Behavioral Sciences Fatima Jinnah Women University, \\ Rawalpindi Pakistan \\ najam_sahar@fjwu.edu.pk (Correspondence Author) \\ Naila Muzaffar \\ Graduate from Behavioral Sciences Department Fatima Jinnah Women University, \\ Rawalpindi Pakistan \\ nyla_muzaffar@hotmail.com
}

\begin{abstract}
The present study explored the role of positive emotions, resilience \& family system in social adjustment in Pakistani adolescents. Through convenient sampling, data collected from 150 adolescents ( 70 boys \& 80 girls).In terms of family system 92 were living in nuclear family while 58 in joint family. Urdu version of Modified Differential Emotions Scale, Resilience Scale and Bell's Adjustment Inventory were used. Statistical analysis revealed the presence of significant positive relationship between positive emotions and resilience $(\mathrm{r}=.39 * *)$ where girls were slightly more resilient $(\mathrm{M} \pm \mathrm{SD}=120.77 \pm 24.03) \&$ socially adjusted $(\mathrm{M} \pm \mathrm{SD}=17.41 \pm 4.15)$. Moreover $52 \%$ adolescents reported difficulty in talking with new people. Majority of participants from Joint family reported good social adjustment (38\%) and high resilience (17\%) as compared to those in nuclear family $(21 \%, 13 \%)$. The influence of these demographic variables is important indication to be explored further.
\end{abstract}

Keyword: Family system, positive emotion, resilience, and social adjusment

\section{Introduction}

The shift from childhood to adolescence is considered to be a challenging period because the individual has to deal with various social, cultural and psychological changes. The entry into the new education system, social circle, and cultural values together with major biological changes can have serious consequences on the social adjustment among college students. There is sufficient literature addressing the psychological distress in adolescents, however, limited data is available on exploring the role of positive emotions in social adjustment according to the culture.

In Pakistani multi-ethnic society, youth comprises of $22 \%$ of the total population which makes it one of the largest pollution of adolescents that can be benefited from the exploration of the relation between psychosocial well-being and cultural dynamics in this changing world. Some researchers (Essau et al. 2012; Kessler et al., 2009) have reported high rates of anxiety \& depression which led to higher intake of anti-depressant \& anti-anxiolytic medications as 
compared to adult population (Bihagen et.al, 2015). Apart from major stressors a lot of daily hassles are present in the life of adolescents such as academic difficulties, conflicts with parents, disagreement with teachers, disappointments with friends and transition from one educational institution to another and due to the high frequency of their occurrence, they have greater impact on individual mental health \& well-being (DuBois, Felner, Brand, Adan, \& Evans, 1992).

The situation is similar in Pakistan. The significant stressors for Pakistani adolescents include academic stress, relationship issues as well as the current sociopolitical situation (Khalily, 2010, 2011). In a study by Mujeeb and Zubair (2012), it was found that survivors of the armed conflict in Pakistan faced many difficulties and psychological trauma after the internal displacement. Those individuals who were highly resilient had less level of depression and anxiety as compared to those who had low resilience. Moreover, it was also found that women were less resilient than men and they were more likely suffering from anxiety and stress (Mujeeb\&Zubair, 2012).

There are some psychological factors that can work as buffers in dealing with these problems. For the present research resilience \& positive emotions have been considered as buffers of social adjustment. According to Vaillant (2008), positive emotions such as gratitude, love, awe, compassion, hope, joy, forgiveness and trust make the individual more creative, efficient, integrative and flexible. Some researchers have suggested that positive emotions enhance the capability of students for learning by increasing motivation, engagement, achievement and interest. Those students who experience more positive emotions are better adapted to the new environment as well as they participate more in the extra-curricular activities (Bigdeli \& Rahimi, 2014; Park, Knorzer, Plass, and Brunken, 2015; Wagnild \& Young, 1993).

Along with these psychological factors, there are some societal contributors towards adjustment of the individual. In a Pakistani research, it was identified that family problems and parental conflicts negatively affect the social adjustment of children (Sabah, Gilani, Kamal, \&Batool, 2012). A number of researchers from Pakistan have assessed role of parents in the adjustment of their children (Akhlaq, Malik, \& Khan, 2013; Hussain \&Munaf, 2012; Khalid, 2004); however there is limited number of published researchers assessing family structure (joint or nuclear) and its impact on the social adjustment and well-being. Pakistani families are mostly patriarchal, collectivistic and large where the elders in the family are making the major decisions about marriage, career \& living styles for all family members. Along with nuclear family, the joint family system is common in Pakistan where two to three generations live together under one roof (Baig, 2014). These families are considered to be close, strong \& resilient with the focus on discipline, obedience, and adherence to norms. This family system provides social \& financial support to its members; however it due to less independence in decision making, adolescents might face problems in personal space, freedom of choice and uniqueness. Therefore it is important to study the influence of family system on psychological well-being and social adjustment of adolescents for prediction of success $\&$ adjustment in future life. 
Rationale of the study

Adolescence is the critical period of life with multiple biological, environmental and social changes which can have an impact on the mental health and social adjustment of adolescents. There is a limited number of published data set from Pakistan assessing the role of positive emotions and psychological resilience in this group. So the present study will contribute in evaluating the role of positive psychology in social well-being of the individual.

\section{Objectives}

To find out the relationship between positive emotions, resilience and social adjustment among college students

To assess the influence of family system on psychological well-being and social adjustment of adolescents

To find out the positivity relation among college students

\section{Method}

The study is a correlation research with a focus on family system influence on psychological well- being regarding positive emotions experience and social adjustment of adolescents.

\section{Participants}

A total of 150 college student (70 boys \& 80 girls, age ranged 16-20) filled the Urdu versions of modified Differential Emotions Scale (mDES), Resilience Scale (RS) and Bell`s Adjustment Inventory (BI) along with the demographic sheet. The data has been collected through convenient sampling from the twin cities of Rawalpindi \& Islamabad.

The following table depicts the frequencies $(f) \&$ percentage $(\%)$ values of the participants. 
Table 1

Frequency distribution, percentage, Mean and Standard deviation table of demographic variables $(N=150)$

\begin{tabular}{|c|c|c|c|}
\hline Variables & Categories & $\mathrm{f}(\%)$ & M (S.D) \\
\hline \multirow[t]{2}{*}{ Gender } & Boys & $70(46.7)$ & \\
\hline & Girls & $80(53.3)$ & \\
\hline \multirow[t]{5}{*}{ Age } & 16 years & $56(37.3)$ & $17.49(1.45)$ \\
\hline & 17 years & $27(18)$ & \\
\hline & 18 years & $25(16.7)$ & \\
\hline & 19 years & $21(14)$ & \\
\hline & 20 years & 21(14) & \\
\hline \multirow[t]{4}{*}{ Class } & High School $\left(9^{\text {th }}\right.$ Grade $)$ & $74(49.3)$ & \\
\hline & High School $\left(10^{\text {th }}\right.$ Grade $)$ & $26(17.3)$ & \\
\hline & Freshman & $25(16.7)$ & \\
\hline & Sophomore & $25(16.7)$ & \\
\hline \multirow[t]{2}{*}{ Family System } & Joint family system & $58(39)$ & \\
\hline & Nuclear system & $92(61)$ & \\
\hline \multirow[t]{4}{*}{ Birth Order } & Eldest child & $48(32)$ & \\
\hline & Middle child & $34(22.7)$ & \\
\hline & Youngest child & $63(42)$ & \\
\hline & Only child & $5(3.3)$ & \\
\hline \multirow[t]{3}{*}{ Residence } & At home & $147(98)$ & \\
\hline & In hostel & $2(1.3)$ & \\
\hline & Others & $1(.7)$ & \\
\hline \multirow[t]{7}{*}{ Share worries with } & No one & $24(16)$ & \\
\hline & Parents & $63(42)$ & \\
\hline & Siblings & $19(12.7)$ & \\
\hline & Friends & $38(25.3)$ & \\
\hline & Significant others & $1(.7)$ & \\
\hline & Others & $4(2.7)$ & \\
\hline & Missing & $1(.7)$ & \\
\hline \multirow[t]{2}{*}{ Significant people in which everything can be shared } & No one & $43(28.7)$ & \\
\hline & Parents & $37(24.7)$ & \\
\hline
\end{tabular}

Instruments

Following instruments were used in the present research.

a. Demographic sheet developed in Urdu consists of open and closed-ended questions related to age, gender, education, the number of siblings, birth order, family system, residence, the number of friends, hobbies (outdoor, indoor), copies mechanism often used, support network.

b. Modified Differential Emotions Scale, (mDES) by Fredrickson (2009) 
Modified Differential Emotions Scale is a reliable \& valid instrument to assess positive and negative emotions separately. It is a 20 items instrument with 5 points Likert scoring. The respondent is asked to think back about the past 24 hours and then give a response against each item. Overall positive and negative emotions ratio can also be created by computing the mean of 10 positive emotions and ten negative emotions.

Translation Procedure: For the current research this scale has been translated into Urdu with the consent of the author. Committee approach was used to translate the scale. Three bilinguals were selected among which one was a psychologist and two were the university professor. Each of them translated the scale separately in Urdu. Those translations were then compared with each other in a meeting. The best translation was chosen and then again given to three different bilinguals for backward translation in English. The most appropriate translated draft was used in the pilot study to check the reliability. Thirty participants were selected for pilot study, and Urdu version of the scale was administered. Further, the reliability of the scale was computed, and it was found that the scale had an alpha reliability of .86, so this translated version was used in this research.

\section{Resilience Scale (RS) by Wagnild \& Young (1993)}

Resilience Scale is a 25 items instrument with 7 points Likert scoring (from strongly agree to strongly disagree) which assesses the individual capability of mediating the adverse effects of negative events. All items are positively scored, and the score ranges from 25 to 175 . The total score can be obtained by adding the responses. A score greater than 145 shows high resilience, score in the range 125 to145 shows a moderate level of resilience and score below 125 shows low levels of resilience. The Urdu version was used in the present research (unpublished thesis by Gulfraz \& Sahar, 2010).

\section{Bell`s Adjustment Inventory, (BI) by Bell (1963)}

The inventory was developed by Bell in 1963 \& extensively used to assess adjustment in four domains (Health, Home, Social and Emotional) in adolescents as well as adults. There are 140 items with dichotomous scoring. High scores on the scale showed more maladjustment while low scores show good adjustment of the person. Home adjustment is expressed in elaborative terms of a person`s satisfaction and dissatisfaction with home life, health adjustment assesses adjustment regarding illness and sickness, Social adjustment measures regarding introversion, extroversion, shyness and submissiveness, etc. while Emotions adjustment is measured regarding nervousness, depression and happiness. The Urdu translation of the Bell's Adjustment Inventory (BI) was used in the present study (Shabbir \& Sahar 2014).

\section{Procedure}

At the first stage, the modified Differential Emotions Scale (mDES) was translated into Urdu. After assessing its reliability, the main study was conducted. The data has been collected in a 
group setting after obtaining the written consent from the participants \& school administration. A brief introduction of the research was given by the researcher herself. During the data collection, the research was present to handle any query. The average time taken by each participant to fill the questionnaire was 30 to 40 minutes. The data collection process was completed in a period of approximately three weeks.

\section{Results}

For the present research, SPSS (v.20.0) has been used for statistical analysis. Descriptive statistics were computed for demographics such as gender, age, family system, birth order, the number of friends, hobbies, etc. Open-ended questions were categorized by previous researches. For example questions about coping mechanism were categorized by categories given by Kausar (2001) for Pakistani population, attribution of failure was categorized by categories given by Weiner (1985) and hobbies were categorized as indoor and outdoor. Pearson correlation and linear regression were computed to assess the role of the family system, positive emotions, and resilience in social adjustment of adolescents.

The sample of present research comprised of 150 adolescents ( $46.7 \%$ boys $/ 53.3 \%$ girls). The results of the demographic variables illustrated that $60.7 \%$ of the participants belonged to a nuclear family system which tells us about the changing trends in the society i.e. shifting from joint family system to nuclear family system in Pakistan. In the demographic sheet, some open questions were added to see their closeness \& disclosure pattern, coping style, sharing of worries and emotional experiences. Most of the participants reported difficulty in decision making (72.7\%) changes in the environment had an effect on $80.7 \%$ of the participants, $52 \%$ of the students in the data experienced problems while talking with strangers. This gives an idea about their daily life pattern and stressors. Since the data set includes more girls, therefore, talking to a stranger has been identified as an important concern due to cultural constrains.

Table 2 represents the correlation values for family system, positive emotions, resilience and social adjustment which indicate a positive relationship between the family system and social adjustment. Furthermore, positive emotions are significantly related to resilience and negative emotions. 
Table 2

Pearson Product Moment Correlation between Family System, Positive emotions (POS), Resilience (RS) and Social Adjustment (SOC) $(N=150)$

\begin{tabular}{lclllll}
\hline Scales/Sub Scales & 1 & 2 & 3 & 4 & 5 & 6 \\
\hline 1. Family System & - & -.02 & .02 & .00 & -.01 & $.21^{*}$ \\
2. $\mathrm{POS}(\mathrm{Ss})$ & - & - & $.19^{*}$ & $.75^{* *}$ & $.37^{* *}$ & .11 \\
3. NEG(Ss) & - & - & - & $.80^{* *}$ & -.07 & $.24 * *$ \\
4. $\mathrm{mDES}$ & - & - & - & - & $.18^{*}$ & $.29^{* *}$ \\
5. RS & - & - & - & - & - & .02 \\
6. SOC $(\mathrm{Ss})$ & - & - & - & - & - & -
\end{tabular}

Note. $\mathrm{mDES}=$ modified differential emotions scale, $\mathrm{POS}=$ positive emotions, $\mathrm{NEG}=$ negative emotions, $\mathrm{RS}=$ resilience scale, $\mathrm{SOC}=$ social domain, $\mathrm{Ss}=$ subscales.

In Table 3 the cross tabulation for social adjustment based on the family system is represented which indicates average to good social adjustment $(n=26)$ by adolescents living in the joint family system as compared to those living in nuclear family setup $(n=18)$.

Table 3

Cross tabulation and Chi - square value for Social Adjustment based on the categories of Family Setup $(N=150)$

\begin{tabular}{|c|c|c|c|c|c|c|c|c|c|}
\hline \multirow{4}{*}{$\begin{array}{l}\text { Categories of } \\
\text { Family Setup }\end{array}$} & \multicolumn{6}{|c|}{ Categories of Social Adjustment } & \multirow[t]{3}{*}{$X^{2}$} & \multirow[t]{4}{*}{$\mathrm{Df}$} & \multirow[t]{3}{*}{$\mathrm{p}$} \\
\hline & Very poor & Poor & Average & Good & Excellent & Total & & & \\
\hline & $(21-40)$ & $(15-20)$ & $(8-14)$ & $(4-7)$ & $(0-3)$ & & & & \\
\hline & $\mathrm{f}(\%)$ & $\mathrm{f}(\%)$ & $\mathrm{f}(\%)$ & $\mathrm{f}(\%)$ & $\mathrm{f}(\%)$ & $\mathrm{f}(\%)$ & & & \\
\hline Joint & $15(17.5)$ & $25(43.9)$ & $21(36.8)$ & $1(1.8)$ & - & $58(100)$ & 8.21 & 6 & .22 \\
\hline Nuclear & $18(19.6)$ & $55(59.8)$ & $19(20.7)$ & - & - & $92(100)$ & & & \\
\hline
\end{tabular}


Table 4 represents the cross tabulation values on resilience based on the family system. Here again, participants from joint family system reported a high level of resilience(17.2\%) as compared to nuclear family $(13.2 \%)$.

Table 4

Cross tabulation and Chi-square value of Resilience-based on the categories of Family setup $(N=$ 150)

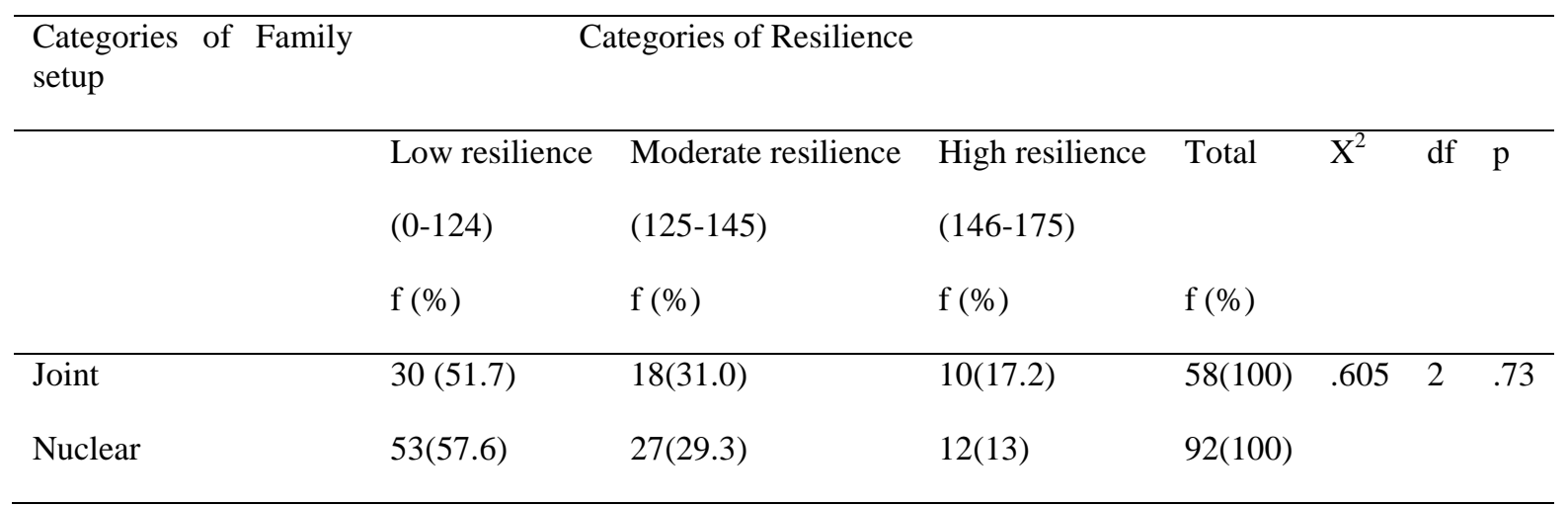

To see the contributing role of positive emotions and resilience, multiple regression analysis has been computed. Table 5 indicates a significant contribution of family system on social adjustment of adolescents $(B=1.64 * *)$ accounting for $20 \%$ variance in social adjustment. Furthermore, negative emotions are found to be a significant contributor in social adjustment rather than positive emotions. This is interesting finding that requires further exploration. 
Table 5

Summary of multiple regression analysis for Positive emotions, Negative emotions, Resilience and Social adjustment $(N=150)$

\begin{tabular}{lcccc}
\hline & & & & Social Adjustment \\
\hline Variables & B & S.E & $\beta$ & $95 \% \mathrm{CL}$ \\
\hline Family system & 1.64 & .65 & $.20^{* *}$ & {$[.38,2.97]$} \\
Positive emotions & .01 & .05 & .02 & {$[-.08, .10]$} \\
Negative emotions & .11 & .04 & $.24^{* *}$ & {$[.04, .19]$} \\
Resilience & .02 & .02 & .11 & {$[-.01, .05]$} \\
$\mathrm{R}$ & .34 & & & \\
$\mathrm{R}^{2}$ & .16 & & & \\
$\mathrm{~F}$ & 4.66 & & & \\
$\triangle \mathrm{R}^{2}$ & .09 & & & \\
$\triangle \mathrm{F}$ & .00 & & & \\
\hline
\end{tabular}

Note. $\mathrm{B}=$ slope, $\mathrm{S} . \mathrm{E}=$ standard error, $\beta=$ standardized beta, $\mathrm{CL}=$ confidence interval, $\mathrm{R}=$ coefficient of correlation. $* * \mathrm{p}<.01$

\section{Discussion}

While analyzing the demographic profile of participants it is interesting to know that majority of participants are living with their parents (98\%), and in the nuclear family system (61\%) which indicates the changing trends of having a small family (nuclear family) in developing countries like Pakistan. There are several reasons for the increase in this shift; it may include inflation, urbanization, education, overpopulation and industrialization. This shift has major implications on family dynamics and child development; some are positive while others are negative. Therefore the present research intended this impact regarding emotional well-being and social adjustment.

In the interviews, the majority of adolescents reported sharing their worries with parents (42\%, this is categories in table one regarding frequency and percentages), which reflects a strong bond between parents and their children. Another reason for sharing with their parents can be the 
living conditions since $98 \%$ of them are residing with their parents, therefore, the proximity can be seen in this data set. Having a problem in decision making is another important issue reported by the participants (73\%), since adolescence is the period of major changes in life, therefore, guidance and support is required in this regard.

The results of the study support the objective that a positive association is present between positive emotions and resilience. These findings are consistent with the previous literature which states that positive emotions play a vital role in developing resilience among individuals in stressful situations (Champion, Compass, Dharamsi, Jaser, \& Reising, 2011). Fredrickson (2013) explained ten positive emotions among which Hope is stated as an important contributor in building resilience which further helps in maintaining psychological well-being (Fredrickson, 2013).

The findings indicate a very weak and non significant association between resilience and social adjustment $(r=.08)$. There are some previous studies which suggest that resilience builds up with age. Late adolescents are more aware of their potentials, so they are more resilient (Caroli \& Sagone, 2013). As the current study included participants from middle adolescents together with the late adolescents, so there is no clear relationship shown with the social adjustment. Moreover, this research is focused on the daily life stressors faced by the adolescents but the previous researches which show the association between resilience and social adjustment are conducted on the survivors of major traumatic events. Such as Stratta et al. (2015) found that resilient individuals can better adjust in their social environment after a natural disaster and develop less PTSD symptoms (Carmassi et al., 2015). Similarly, it was found that patients with burns can adjust better in the social environment if they accept the disability and remain resilient to the stressful events (Cheng et al., 2014). Since none of the participants has reported a significant traumatic event in recent years, therefore it can attribute that routine functions may not increase resilience.

A significant relation was reported in the family system and social adjustment. Table 3 shows that participants from the joint family system are better adjusted that those living in a nuclear family. Furthermore, the regression analysis shows $20 \%$ contribution of the family system in social adjustment. These results are consistent with previous researches. In a Pakistani research by Bilal et.al (2013) 60\% of girls belonging to joint family reported emotional stability as compared to $45 \%$ from nuclear family set up. Since the collectivistic culture of Pakistan promotes strong family ties and connectedness, therefore individual life is heavily influenced by family support and acceptance in major spheres of life. The joint family system serves as a support network, provides peer interaction, cooperation as well as a challenge to test individual abilities. This help in social adjustment with increased competence, communication and coordination. In the joint family system, there are more members who provide more chances of disclosure of emotions, number of adults advising in major life issues such as intimacy, parenting, career choices, and selection of academic institutions for adolescents. 
Table $3 \& 4$ demonstrate that participants living in joint family setup have better levels of social adjustment and high resilience as compared to those living in nuclear setups. $55.3 \%$ individuals have a low resilience which can be supported by viewing the demographic variables that $54.1 \%$ of the participants show internal attribution of failure and defeat. Research suggested that lack of optimism, self-criticism, and negative perception are related to low levels of resilience in adolescence (Beesdo, Pine, \& Lieb, 2010). As the majority of the participants show internal attribution of failure and defeat which can later lead towards self-criticism and negative perception, hence they have low levels of resilience.

\section{Conclusion}

This present study is a little effort in understating the role of family setup and emotional wellbeing in individual's life. Findings have identified a strong role of the family system in social adjustment but not the positive emotions and resilience. Family plays a vital role in child development; therefore it is important to assess the impact of family structure on psychosocial well-being regarding Health, interpersonal relations, emotional expression and stress management. Due to globalization and technological advancement, family dynamics\& roles are changing in Pakistani society, so it is important to understand these dynamics for better adjustment of individual and prosperity of society.

\section{References}

Akhlaq,A., Malik,N.I. \& Khan, N.A.(2013). Family Communication and Family System as the Predictors of Family Satisfaction in Adolescents. Science Journal of Psychology,2013.06.doi:10.7237/sjpsych/258

Baig, Noor-ul-Ain., Rehman, Rana Rashid \& Mobeen Noor (2014). A Parent-Teacher View of Teens Behaviors in Nuclear and Joint Family Systems in Pakistan. The Qualitative Report 2014 19, 67, pg 1-12 http://www.nova.edu/ssss/QR/QR19/baig67.pdf

Beesdo, K., Pine, D.S., \& Lieb, R.( 2010). Incidence and risk patterns of anxiety and Categorization of Generalized Anxiety Disorder. Arch Gen Psychiatry.67(1):47-57. doi:10.1001/archgenpsychiatry.2009.177

Bigdeli. R.A.,\& Rahimi, A. (2014). The broad and build theory of positive emotions in second language learning. Procedia - Social and Behavioral Sciences, 159, $795-801$. Retrieved from http://creativecommons.org./liscences/by-nc-nd/3.0/

Bihagen, E., Fall, K., Hiyoshi, A., Montgomery, S., Osika, W., \& Udumyan, R. (2015). Stress resilience in adolescence and subsequent antidepressant and anxiolytic medication in 
middle aged men: Swedish cohort study. Social Science and Medicine, 134, 43-49. Doi: 10.1016/j.socscimed.2015.03.057

Bilal ,Hafiz Ahmad ., Tariq, Abdur Rehman., Aleem, Umaira., Shabbir, Syeda Iqra., \& Parveen, Musarrat(2013). The Effect Of Nuclear And Joint Family Systems On Academic Achievements Of Students. Academic Research International. 4(5).

Caroli, M.E.D., \& Sagone , E. (2013). Relationship between psychological well-being and resilience in middle and late adolescents. Procedia - Social and Behavioral Sciences, 141, 881 - 887. Doi: 10.1016/j.sbspro.2014.05.154

Champion, J.E., Comapss, B.E., Dharamsi, K.R., Jaser, S.S., \& Reising, M.M. (2011). Coping and positive affect in adolescent of mothers with and without a history of depression. Journal of Child and Family Studies, 20(3), 353-360. doi: 10.1007/s10826-010-9399-y

Cheng, Y.H., Huang, R., Kong, Y., Shi, S.H., Xia, Z.Y.,\& Yin, T.T. (2014). The impact of acceptance of disability and psychological resilience on post traumatic stress disorder in burn patients. International Journal of Nursing Sciences, 1, 371-375. Doi: 10.1016/j.ijnss.2014.10.018

DuBois, D. L., Felner, R. D., Brand, S., Adan, A. M., \& Evans, E. G. (1992). A prospective study of life stress, social support, and adaptation in early adolescence. Child Development, 63, 542-557. DOI: 10.1111/j.1467-8624.1992.tb01645.x

Essau, C. A., Leung, P. W., Koydemir, S., Sasagawa, S., O'Callaghan, J., \& Bray, D. (2012). The impact of self-construals and perceived social norms on social anxiety in young adults: a cross-cultural comparison. International Journal of Culture and Mental Health, 5(2), $109-120$

Fredrickson, B. L. (2009). Positivity. New York, NY: Crown Publishers

Fredrickson, B.L. (2013). Positive emotions broad and build. Advances in Experimental Social Psychology, 47. doi.org/10.1016/B978-0-12-407236-7.00001-2

Gen Psychiatry, 67, 47-57. doi:10.1001/archgenpsychiatry.2009.177.

Gulfraz, Sumiha., \& Sahar, Najam-us. (2010). Gender Differences in Resilience and Life Satisfaction among Handicaps. (unpublished master`s thesis). Fatima Jinnah Women University, Rawalpindi.

Hussain, Sadiq., \& Munaf, Seema. (2012).Perceived father acceptance-rejection in childhood and psychological adjustment in adulthood.International ournal of Business and Social Science. 3(1), 149-156. Retrieved from http: // www. ijbssnet. com/ journals/ Vol_3_No_1_January_2012/16.pdf 
Kessler RC, Aguilar-Gaxiola S, Alonso J, Chatterji S, Lee S, Ormel J, Ustün TB, Wang PS. The global burden of mental disorders: an update from the WHO World Mental Health (WMH) surveys. Epidemiol Psichiatr Soc. 2009;18(1):23-33.

Khalid, S. (2004). Development and validation of the index of personal growth and the familial and disposition al predictors of personal growth (Thesis of $\mathrm{PhD}$ ) National Institute of Psychology and centre of Excellence, Quaid-e-Azam University: Islamabad.

Khalily, M.T.,(2010). Developing an integrated approach to the mental health issues in Pakistan. Journal of Inter-professional Care. 24(2):168-72

Mujeeb, A. \& Zubair, A.(2012). Resilience, stress, anxiety and depression among internally displaced persons affected by armed conflict. Pakistan Journal of Social and Clinical Psychology, 10(2), 20-26. Retrieved from http://www.gcu.edu.pk/Soc\&ClinPsyJour.htm

Park , B., Knorzer, L., Plass, J. L., \& Brunken, R. (2015). Emotional design and positive emotions in multimedia learning: an eye tracking study on the use of anthropomorphisms. Computers and Education, 86, 30-42. doi.org/10.1016/j.compedu.2015.02.016

Sabah , S., Gilani, N., Kamal. A., \& Batool, S. (2012). Chaotic home conditions and children`s adjustment: a study of gender differences. Pakistan Journal of Psychological Research, 27(2), 297-313. Retrieved from http://www.pjprnip.edu.pk/pjpr/index.php/pjpr

Shabbir, Neelum., \& Sahar, Najam-us (2014). Role of Emotional Resilience in Social Adjustment of Young Adults: A Pilot Study. (unpublished master`s thesis). Fatima Jinnah Women University, Rawalpindi.

Stratta, Paolo., Capanna, Cristina., Dell’Osso, Liliana., Carmassi, Claudia., Patriarca, Sara., Di Emidio, Gabriella., Riccardi, Ilaria., Collazzoni, Alberto \& Rossi, Alessandro (2015). Resilience and coping in trauma spectrum symptoms prediction: a structural equation modeling approach. Personality and Individual Differences, 77, 55-61. Doi: 10.1016/j.paid.2014.12.035.

Vaillant, G.E. (2008). Positive emotins, spirituality and the practice of psychiatry. Mental Health, Spirtuality, Mind, 6(1), 48-62. DOI: 10.4103/0973-1229.36504

Wagnild, G.M. \& Young, H.M. (1993). Development and psychometric evaluation o resilience scale. Journal of Nursing Measurement, 1(2). Retrieved from http://www.ingentaconnect.com/content/springer/nmeas 